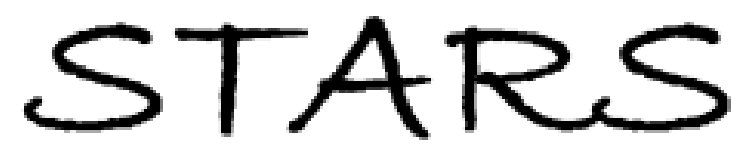

University of Central Florida

STARS

$1-1-2007$

\title{
Thermal conductance across grain boundaries in diamond from molecular dynamics simulation
}

Taku Watanabe

Boris $\mathrm{Ni}$

Simon R. Phillpot

Patrick K. Schelling

University of Central Florida

Pawel Keblinski

Find similar works at: https://stars.library.ucf.edu/facultybib2000

University of Central Florida Libraries http://library.ucf.edu

This Article is brought to you for free and open access by the Faculty Bibliography at STARS. It has been accepted for inclusion in Faculty Bibliography 2000 s by an authorized administrator of STARS. For more information, please contactSTARS@ucf.edu.

\section{Recommended Citation}

Watanabe, Taku; Ni, Boris; Phillpot, Simon R.; Schelling, Patrick K.; and Keblinski, Pawel, "Thermal conductance across grain boundaries in diamond from molecular dynamics simulation" (2007). Faculty Bibliography 2000s. 7778.

https://stars.library.ucf.edu/facultybib2000/7778

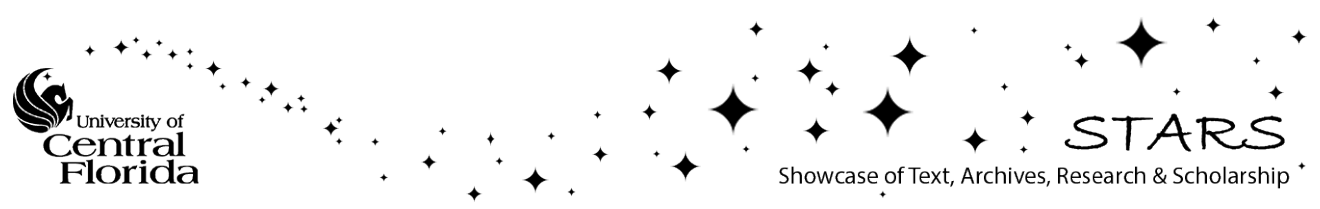




\section{Thermal conductance across grain boundaries in diamond from molecular dynamics simulation}

Cite as: J. Appl. Phys. 102, 063503 (2007); https://doi.org/10.1063/1.2779289

Submitted: 13 May 2007 . Accepted: 25 July 2007 . Published Online: 17 September 2007

Taku Watanabe, Boris Ni, Simon R. Phillpot, Patrick K. Schelling, and Pawel Keblinski

\section{ARTICLES YOU MAY BE INTERESTED IN}

Kapitza conductance and phonon scattering at grain boundaries by simulation Journal of Applied Physics 95, 6082 (2004); https://doi.org/10.1063/1.1702100

Thermal transport and grain boundary conductance in ultrananocrystalline diamond thin films Journal of Applied Physics 99, 114301 (2006); https://doi.org/10.1063/1.2199974

Nanoscale thermal transport

Journal of Applied Physics 93, 793 (2003); https://doi.org/10.1063/1.1524305

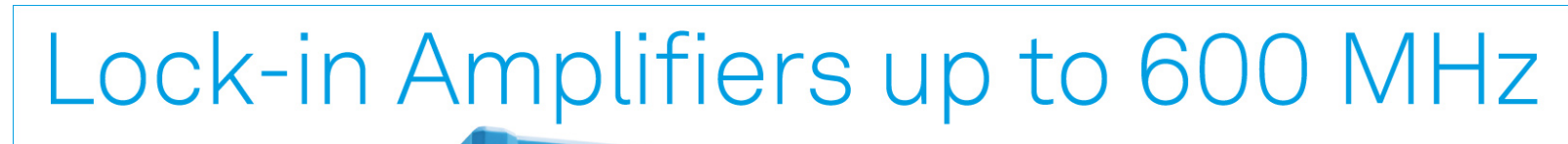

starting at $\$ 6,210$

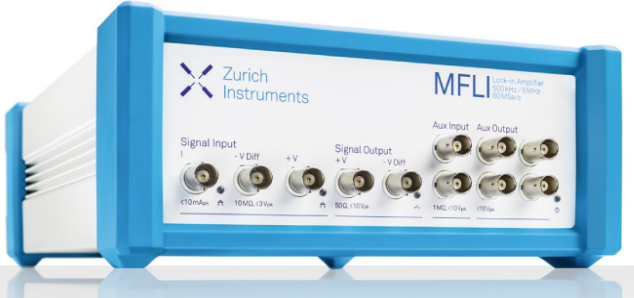

J. Appl. Phys. 102, 063503 (2007); https://doi.org/10.1063/1.2779289

(c) 2007 American Institute of Physics. 


\title{
Thermal conductance across grain boundaries in diamond from molecular dynamics simulation
}

\author{
Taku Watanabe, Boris Ni, and Simon R. Phillpot ${ }^{\mathrm{a})}$ \\ Department of Materials Science and Engineering, University of Florida, Gainesville, Florida 32611, USA
}

Patrick K. Schelling

Advanced Materials Processing and Analysis Center, University of Central Florida, Orlando, Florida 32816, USA and Department of Physics, University of Central Florida, Orlando, Florida 32816, USA

Pawel Keblinski

Department of Materials Science and Engineering, Rensselaer Polytechnic Institute, Troy, New York 12180, USA

(Received 13 May 2007; accepted 25 July 2007; published online 17 September 2007)

\begin{abstract}
We determine the dependence of the interfacial conductance on twist angle for (001) symmetric twist grain boundaries (GBs) in diamond. We find that the conductances are extremely large, ranging from 7.7 to $17.6 \mathrm{GW} / \mathrm{m}^{2} \mathrm{~K}$. Nevertheless, when normalized to the single-crystal conductivity, the resulting Kapitza lengths are actually longer in diamond than in Si, indicating that the diamond GBs are relatively worse conductors of heat. This result is consistent with the poorer bonding across the diamond grain boundaries. We find that the interfacial conductance and Kapitza length can be well fitted by an extended Read-Shockley model. (C) 2007 American Institute of Physics.
\end{abstract}

[DOI: $10.1063 / 1.2779289]$

\section{INTRODUCTION}

The relentless drive to faster and smaller microelectronics is leading to dramatic increases in the thermal load on functional nanostructures. ${ }^{1}$ A detailed understanding of thermal transport in such nanostructures is therefore imperative. As system sizes decrease to nanometer scales, the dominant phonon scattering mechanism changes. For relatively largegrain sized materials, phonon-phonon and phonon-point defect scatterings are the dominant mechanisms. However, the dimensions of nanostructures are becoming of the order of, or even smaller than the phonon mean free path $(\sim 150 \mathrm{~nm}$ in diamond at room temperature, shorter in other materials). As a result, while phonon-point defect interactions remain important, phonon-phonon interactions become relatively less important. On these length scales, the scattering of phonons from the microstructure, particularly surfaces and grain boundaries (GBs), becomes a key process in determining the effectiveness of heat flow. ${ }^{2}$

Since the experimental investigation of thermal transport at individual interfaces is extremely difficult, there is an excellent opportunity for simulation to provide important insights. Indeed, over the last few years, there has been considerable activity in simulating heat transport both in traditional materials and in nanodimensioned materials. For example, the effect of nanodimensions has been explored in some detail in simulations of thermal transport in superlattices $^{2}$ and, more recently, in nanowires. ${ }^{3,4}$ While there have been simulations of some individual grain boundaries, ${ }^{5,6}$ no study has systematically explored the effect of the structure of the interface itself on thermal transport. In

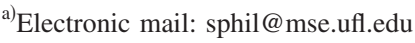

this paper, we elucidate how the interfacial conductance changes with the crystallography of the grain boundary. Diamond is an ideal laboratory in which to characterize these effects since it has the largest thermal conductivity of any material; thus, the detrimental effects of the grain boundaries can be expected to be particularly evident. In addition, diamond is of considerable technological interest. ${ }^{6-9}$ From our simulations, we find that the interfacial conductance is extremely high; however, when appropriately normalized, it is less than that of the corresponding grain boundaries in silicon, a result that we can understand in terms of the bonding at the interfaces. Moreover, we find that the interfacial resistance is an approximately linear function of the GB energy. As a consequence, we find that it is possible to construct a Read-Shockley type model for the thermal conductance of these boundaries.

\section{SIMULATION METHODS}

In this work, diamond is simulated using the potential by Tersoff, ${ }^{10}$ a well-known and well-characterized many-body potential for covalently bonded materials such as $\mathrm{C}$, $\mathrm{Si}$, and Ge. The Tersoff potential works particularly well for carbon because of its capability to describe both $s p^{2}$ and $s p^{3}$ hybridizations.

The thermal conductivity simulations have been performed using the direct method by Jund and Jullien. ${ }^{11}$ As Fig. 1 shows, and as has been discussed in detail elsewhere, ${ }^{5}$ in the direct method, a small region of the bulk is heated to act as the heat source, while another region is cooled to act as the heat sink. A fixed amount of energy is removed from the heat sink region and an identical amount of energy is added in the heat-source region; thus, the total energy of the system is conserved. 


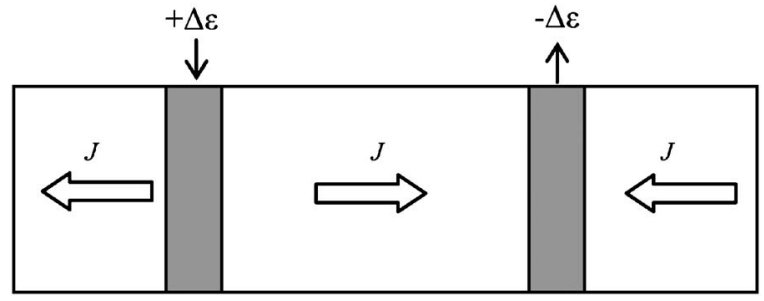

FIG. 1. (top) Simulation cell setup for the direct method for simulating thermal conductivity. The heat source and sink are located at a quarter of the cell length away from the center of the cell. The same amount of energy $\Delta \varepsilon$ is added to the atoms in the heat source and removed from those in the heat sink. This setup results in two equivalent heat currents $J$ in opposite directions along the $z$ axis.

For a spatially homogeneous system, such as a perfect crystal, this process results in a thermal gradient $d T / d x$ being set up in the system. The thermal conductivity $\kappa$ can then be calculated from Fourier's law,

$$
J=-\kappa d T / d x
$$

where $J$ is the heat current density. In general, $\kappa$ is a ranktwo tensor; however, it is treated as a scalar in the case of one-dimensional heat flow as in our simulations.

For a system containing a grain boundary, the interfacial thermal conductance $G_{K}$ can be calculated in a similar fashion from the temperature drop $\Delta T$ across the interface,

$$
J=G_{K} \Delta T .
$$

This method was also used in our earlier study of $\mathrm{Si}$ grain boundaries and was shown to work. ${ }^{12}$

\section{INTERFACIAL CONDUCTANCE OF (001) TWIST GRAIN BOUNDARIES}

The diamond crystal structure is characterized by four covalent bonds pointing along the $\langle 111\rangle$ directions. A grain boundary on an arbitrary plane will thus have two covalent bonds projecting back into the grain itself and two bonds across the grain boundary. ${ }^{13}$ These latter two bonds can be expected to be significantly different from the bonds in the bulk due to the structural disorder at the grain boundary. If we examine the principal planes in the diamond lattice, grain boundaries on the (111) plane have three bonds back into the crystal and one across the interface; grain boundaries on the (011) plane have one bond pointing back into the crystal, two parallel to the interface, and one pointing across the interface. Neither of these is similar to the typical grain boundary described above. However, grain boundaries on the (001) plane have two bonds pointing back into the crystal and two pointing across the interface. A high-angle twist boundary on the (001) plane may thus be considered as being, to a considerable extent, representative of the grain boundaries in polycrystalline and nanocrystalline diamond. ${ }^{13}$ Therefore, to elucidate the structure-property relationship for a generic grain boundary in diamond, we determine how the thermal conductance of (001) GBs varies with twist angle.

The diamond lattice has a twofold symmetry axis in the $\langle 001\rangle$ direction. Thus, on forming symmetric twist boundaries on this plane, a rotation of one semicrystal with respect to the other by $180^{\circ}$ will result in a single crystal. Therefore, to probe the full range of grain boundaries, we only need to consider twist angles from $0^{\circ}$ to $90^{\circ}$. We have simulated the thermal-transport properties of 12 such GBs, with twist angles ranging from $8.80^{\circ}$ to $90.0^{\circ}$. These GBs were formed by cutting a single crystal along an (001) plane and rotating one-half of the crystal with respect to the other. The unit cells for the GBs considered range in areal dimensions from being equal to that of the perfect crystal $\left(\theta=90^{\circ}\right.$ and $\left.\Sigma=1\right)$ to being 101 times larger $\left(\theta=11.42^{\circ}\right.$ and $\left.\Sigma=101\right)$.

The $90^{\circ} \mathrm{GB}$ is a special case and is actually a symmetric twin GB. In the $\langle 001\rangle$ direction, the stacking of the perfect crystal can be written as $\cdots A a B b A a B b A b \cdots$, where the $A a$ layers come from the fcc Bravais lattice with a basis of two atoms at $(0,0,0)$ and $(0.25,0.25,0.25)$. The $90^{\circ}$ twist GB then corresponds to a stacking sequence of $\cdots A a B b A b B a A b \cdots$, in which we can see that the stacking sequence is symmetric about one of the $A$ planes. Full crystallographic details of all of the GBs considered and a summary of the simulation results are given in Table I.

To prepare the GBs for the thermal conductivity simulations, the unrelaxed GB structures were heated to $2000 \mathrm{~K}$ to minimize the stress, then slowly cooled to near $0 \mathrm{~K}$ in a stepwise fashion; equilibrium $T=0 \mathrm{~K}$ structures were then determined by steepest descent quenches to minimize the energies and to reduce the forces and stresses to negligible values. This process was determined to be appropriate in an earlier work. ${ }^{13}$ A detailed analysis showed that the coordination of atoms within the typical high-angle high-energy GB, $\Sigma 29(001)$, agrees very well with our previous results. ${ }^{13}$ To minimize the computer time, the relaxation process was performed on simulation cells that were only 16 unit cell long. After the relaxed structure was obtained, perfect crystal regions were inserted to form structures long enough in the $z$ direction for the thermal conductivity simulations. von Alfthan et al. ${ }^{14}$ investigated the zero-temperature structures of Si GB structures by removing atoms from the GB region and found GB structures with energies lower than what was previously reported. We have explored this effect by performing the same procedure on a $\Sigma 29$ (001) GB in diamond, but we did not find any structures with energies lower than those formed by the process described above. The $\Sigma 29(001)$ GB has one of the highest energies and is thus one of the most likely to undergo reconstruction since the potential energy saving is the largest. Because our simulations on this grain boundary showed no reconstruction, it is unlikely that the other, lower energy grain boundaries will lower their energies by reconstruction.

Before discussing the dependence of the thermaltransport properties on the twist angle, we examine the representative case of the (001) $\theta=43.60^{\circ}(\Sigma 29)$ GB. Because the simulation is set up as a three-dimensional (3D) periodic system with crystallographically identical GBs at the center of the unit cell and at the edge, the heat source and heat sink are equidistant between the two grain boundaries. We performed seven test runs with heat current densities ranging from $1 \times 10^{-4}$ and $8 \times 10^{-4} \mathrm{eV} / \mathrm{nm}^{2}$ per MD step. The maximum difference in the conductivity values was $10 \%$ and there was no systematic trend. A similar weak dependence on the heat flux was observed in our previous work on Si GBs. ${ }^{12}$ 
TABLE I. Summary of the diamond (001) GB properties. $\Sigma$ indicates the size of the coincident site lattice (CSL) planar unit cell of the GB. $\theta$ is the twist angle in deg, $E_{\mathrm{GB}}$ is the grain boundary energy, and $\delta V$ is the volume expansion in units of lattice parameter $a_{0}$. $G_{K}$ is the calculated thermal conductance, $l_{K}$ is the Kapitza length defined by $l_{K}=\kappa / G_{K}$, where $\kappa$ is the thermal conductivity of the perfect crystal diamond. $\langle C\rangle$ is the average coordination number of the atoms within the GBs, and $C_{n}$ is the fraction of $n$ coordinated atoms within the GBs.

\begin{tabular}{rccccccccc}
\hline \hline$\Sigma$ & $\begin{array}{c}\theta \\
(\mathrm{deg})\end{array}$ & $\begin{array}{c}E_{\mathrm{GB}} \\
\left(\mathrm{J} / \mathrm{m}^{2}\right)\end{array}$ & $\begin{array}{c}\delta V \\
\left(a_{0}\right)\end{array}$ & $\begin{array}{c}G_{K} \\
\left(\mathrm{GW} / \mathrm{m}^{2} \mathrm{~K}\right)\end{array}$ & $\begin{array}{c}l_{K} \\
\left(a_{0}\right)\end{array}$ & $\begin{array}{c}\langle C\rangle \\
(-)\end{array}$ & $\begin{array}{c}C_{2} \\
(\%)\end{array}$ & $\begin{array}{c}C_{3} \\
(\%)\end{array}$ & $\begin{array}{c}C_{4} \\
(\%)\end{array}$ \\
\hline 85 & 8.80 & 3.66 & 0.28 & 17.6 & 75.9 & 3.24 & 0.00 & 76.5 & 23.46 \\
101 & 11.42 & 4.34 & 0.30 & 13.3 & 100.3 & 3.19 & 0.48 & 80.2 & 19.33 \\
41 & 12.68 & 4.18 & 0.30 & 13.3 & 100.3 & 3.17 & 0.57 & 82.4 & 17.05 \\
25 & 16.26 & 4.87 & 0.31 & 13.9 & 96.0 & 3.15 & 0.97 & 83.5 & 15.53 \\
13 & 22.62 & 5.10 & 0.32 & 11.2 & 119.7 & 3.12 & 2.33 & 83.7 & 13.95 \\
17 & 28.07 & 5.36 & 0.35 & 10.1 & 132.8 & 3.13 & 0.00 & 87.1 & 12.86 \\
5 & 36.87 & 5.84 & 0.38 & 11.7 & 113.7 & 3.12 & 0.00 & 87.8 & 12.2 \\
29 & 43.60 & 6.19 & 0.37 & 8.8 & 151.2 & 3.11 & 3.31 & 82.6 & 14.05 \\
5 & 53.13 & 5.98 & 0.36 & 8.4 & 157.8 & 3.18 & 1.25 & 80.0 & 18.75 \\
17 & 61.93 & 6.38 & 0.38 & 7.6 & 174.2 & 3.18 & 1.47 & 85.3 & 13.24 \\
25 & 73.74 & 5.21 & 0.31 & 9.9 & 135.0 & 3.21 & 0.00 & 78.9 & 21.09 \\
1 & 90.00 & 2.60 & 0.24 & 10.9 & 122.4 & 3.17 & 1.56 & 79.7 & 18.75 \\
\hline \hline
\end{tabular}

Moreover, our previous studies ${ }^{12}$ for perfect crystals showed quantitative agreement for the predicted thermal conductivity between the imposed heat current method and a Green-Kubo approach, in which there is no heat current at all. The heat current density for subsequent simulations was kept constant at $2.5 \times 10^{-3} \mathrm{eV} / \mathrm{nm}^{2} \mathrm{fs}$. The resulting temperature profile in Fig. 2 clearly shows the symmetry of the simulation setup.

In our simulation, temperature at each location in the simulation cell is defined by the average kinetic energy per atomic plane averaged over the last $560 \mathrm{ps}$. The temperature drop across a GB is determined in the following manner. (i) The temperature profiles in the bulk regions surrounding the interface are fitted linearly, with the range of $z$ values determined by minimizing the difference between the absolute values of the slopes. The linear fits are made over the region between 12 and $25 \mathrm{~nm}$, depending on the size of the simula-

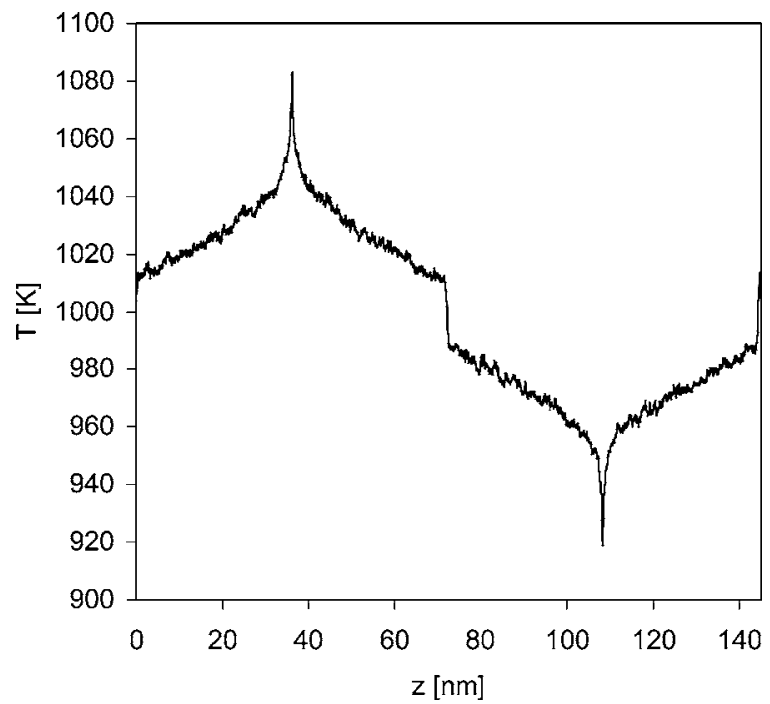

FIG. 2. A typical temperature profile of a system with grain boundaries, in this case, (001) $\theta=43.60^{\circ} \Sigma 29$ symmetric twist boundaries. The grain boundaries are located at the center and the edge of the simulation cell. The average temperature drop across the grain boundaries is $22.5 \mathrm{~K}$. tion cell. The regions are considered bulk when $r^{2}$ of the fit is above 0.95 . Because of the symmetry of the structure, all the slopes are found to be the same within small error bars. (ii) The temperature jumps are determined from the equations to the linear fits. Analysis from the linear fits yields the values of 21.1 and $23.8 \mathrm{~K}$ for the temperature drops at the two crystallographically identical grain boundaries. The average temperature drop from these two GBs is determined to be $22.5 \mathrm{~K}$, and the corresponding thermal conductance is $8.8 \pm 0.6 \mathrm{GW} / \mathrm{m}^{2} \mathrm{~K}$, with the estimated uncertainty in the conductance coming from the difference in the two temperature drops.

The size of the simulation cell has a considerable effect on the calculated thermal conductivity of perfect crystals. ${ }^{12,15}$ The origin of this effect is the restriction on the maximum phonon mean free path set by the length of the periodic simulation cell.

To explore the possibility of an analogous size effect for the interface conductance, we have determined the temperature drop at the interface and the corresponding interfacial conductance for the $\Sigma 29 \mathrm{~GB}$ for simulation cells ranging from 14.3 to $287 \mathrm{~nm}$ in length. As Fig. 3 shows, there is a strong system size dependence; however, it appears that $\Delta T$ reaches an asymptotic value. To confirm this, we have fitted these data by a shifted exponential,

$$
\Delta T=\Delta T_{\infty}+A \exp \left(-L z / L_{0}\right) .
$$

Figure 3 shows that this functional form fits the data well. The values of parameters $A$ and $L_{0}$ obtained from the fit are $60.9 \mathrm{~K}$ and $70.7 \mathrm{~nm}$, respectively. The formal description of the Kapitza conductance is given by Stoner and Maris ${ }^{16}$ as an integration over the wave vector summed over all the branches. Due to the complexity of phonon density of states and dependence of transmission coefficients as a function of dispersion relation, extracting the analytical expression of the size dependence is not straightforward. At present, we do not have a theoretical argument for this functional form but simply justify its use a posteriori by the quality of the fit 


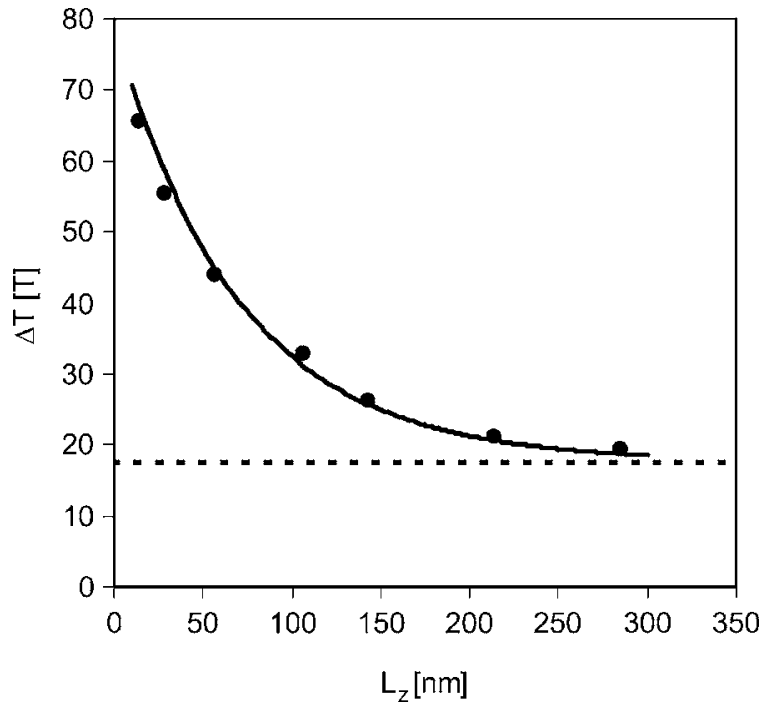

FIG. 3. Size dependence of the thermal conductance of the $\Sigma 29 \theta$ $=43.60^{\circ} \mathrm{GB}$ fitted to a shifted exponential. $\Delta T_{\infty}$ is the asymptotic temperature drop in the infinite size limit indicated by the dotted line.

shown in Fig. 3. The infinite limit temperature drop $\Delta T_{\infty}$ is 17.6 K. The calculated interfacial conductances show a corresponding increase with an asymptotic value of $12.3 \mathrm{GW} / \mathrm{m}^{2} \mathrm{~K}$. Interestingly, we saw no such effect in our previous (albeit less extensive) simulations of this effect for $\mathrm{Si} \mathrm{GBs} ;{ }^{5}$ however, the thermal conductivity of single-crystal $\mathrm{Si}$ is much lower than that of single-crystal diamond and the bonding of atoms at the interfaces is quite different, as will be shown later.

Because the simulations for the longer systems are extremely computer-time intensive, for our systematic studies of the effects of crystallography, we have used a unit cell of $142.4 \mathrm{~nm}$ (400 unit cells) in length. The values of the interfacial conductances discussed below are thus $\sim 30 \%$ lower than the asymptotic values we would predict for a simulation cell of infinite length.

In addition to effects of the length of the simulation cell, we have also considered the effect of its cross section. In the previous study on Si single crystal, the dependence on the width of simulation cell was found to be very weak beyond $3 \times 3$ unit cells for the cross section. ${ }^{12}$ The periodic planar unit cell is $\Sigma$ times larger for GBs than for the single crystal as characterized. As a result, the smallest cross sectional area is $\sim 3.5 \times 3.5$ square units, with most of the simulation cells have larger cross sectional areas.

For the case of the $\Sigma 29 \mathrm{~GB}$, we have also determined the temperature dependence of the interfacial conductance. As Fig. 4 shows, the interfacial conductance increases almost linearly with the temperature up to $1250 \mathrm{~K}$, above which it decreases. This increase is in strong contrast to the bulk thermal conductivity which decreases strongly with temperature. We can understand this in a qualitative manner as arising from the significant obstacle that the grain boundary offers to heat transport. As such, there is poor coupling of the phonon modes on opposite sides of the interface. As the temperature increases, the anharmonicity of the system increases, which increases the coupling of previously weakly coupled phonon modes across the interfaces, thereby enhancing the thermal

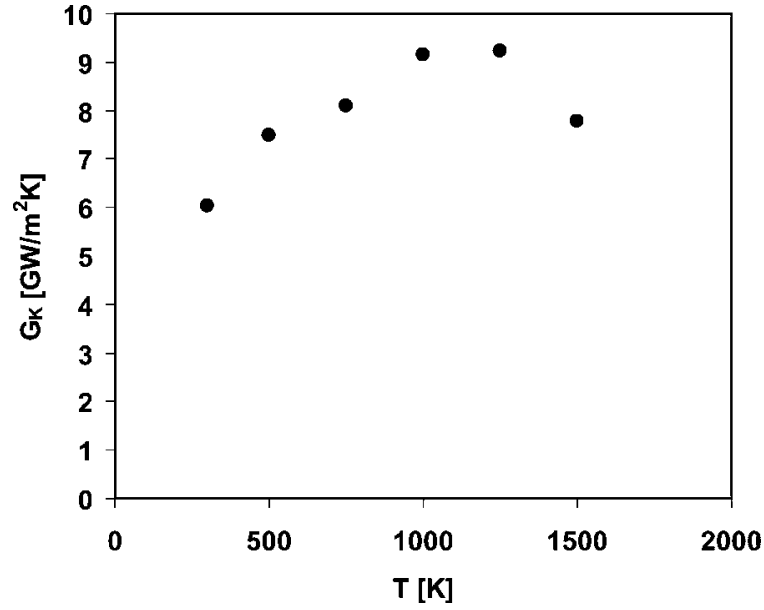

FIG. 4. Temperature dependence of the thermal conductance of the $\Sigma 29 \theta$ $=43.60^{\circ} \mathrm{GB}$. The conductance increases almost linearly with the temperature up to about $1250 \mathrm{~K}$. The drop at $1500 \mathrm{~K}$ is due to the change in structure.

transport. This increase with temperature is consistent with experiments and simulation on a wide range of heterointerfaces. ${ }^{2,17,18}$ Detailed analysis shows that the drop in the conductance at $1500 \mathrm{~K}$ is due to the structural change at the interface, rather than a change in the thermal-transport mechanism itself.

\section{INTERFACIAL THERMAL CONDUCTANCE: DIAMOND VERSUS SILICON}

To determine the effect of crystallography on the interfacial conductance, Fig. 5 shows the calculated thermal conductances at $1000 \mathrm{~K}$ of the twelve diamond grain boundaries as a function of the twist angle for a simulation cell length of $142.4 \mathrm{~nm}$. The interfacial conductance decreases from about $17 \mathrm{GW} / \mathrm{m}^{2} \mathrm{~K}$ for low twist angles to $9 \mathrm{GW} / \mathrm{m}^{2} \mathrm{~K}$ for $\theta$ $=43.6^{\circ}$, before increasing again to $12 \mathrm{~W} / \mathrm{m}^{2} \mathrm{~K}$ for the $90^{\circ} \Sigma 1$ grain boundary. The uncertainties in the calculated values are represented by the error bars, which are typically $\pm 1.3 \mathrm{GW} / \mathrm{m}^{2} \mathrm{~K}$.

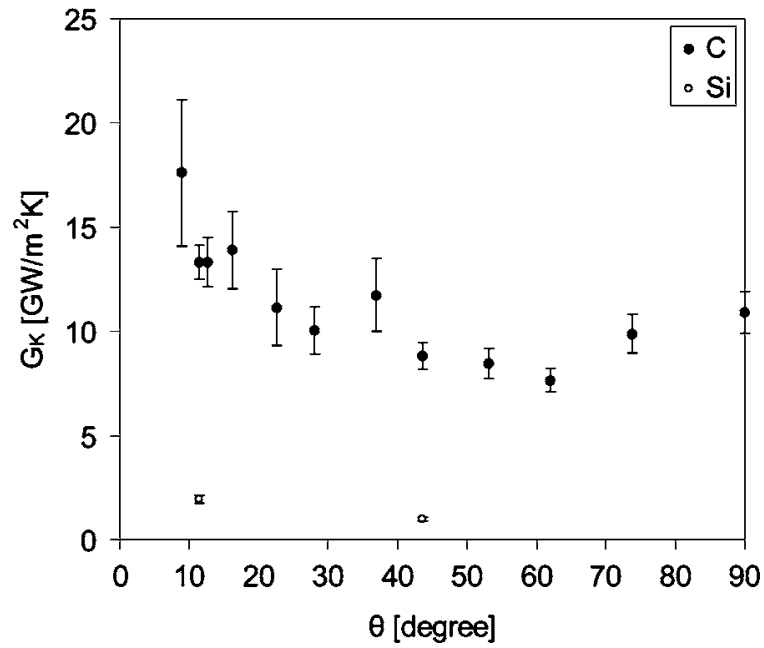

FIG. 5. Thermal conductance as a function of twist angle for diamond (001) symmetric twist grain boundaries. The values determined previously for two Si GBs are also shown. 


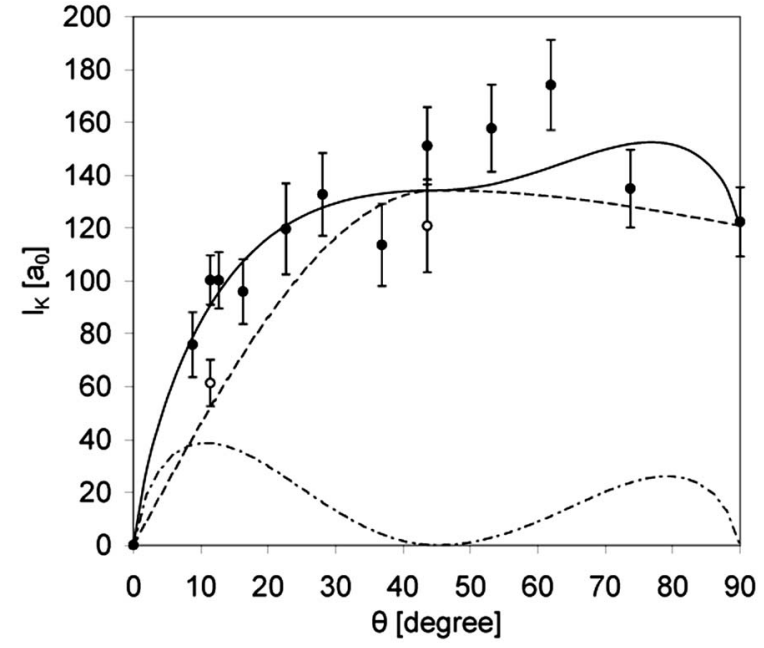

FIG. 6. Kapitza lengths in units of their respective lattice parameters as a function of twist angle for both diamond and Si. Filled circles $(\bullet)$ are for diamond, and the open circles $(\bigcirc)$ are for Si. The solid line is the extended Read-Shockley fit to the diamond data. The dashed line is the dislocation core contribution, while the dash-dot line is the strain field contribution. Both points for Si are about $30 a_{0}$ lower than diamond.

This significant structural dependence, which we analyze in detail below, is quite reasonable since the degree of structural disorder at the GB, for which the energy is a measure, is lowest at small and high twist angles, for which dislocation models of grain boundaries are appropriate, and highest at intermediate twist angles.

In an earlier paper on structure and mechanical behavior, ${ }^{13}$ we found that a comparison of the structure and properties of diamond and silicon grain boundaries to be quite instructive. In particular, diamond readily forms $s p^{2}$ graphitic bonding at grain boundaries, while Si strongly favors the retention of $s p^{3}$ bonding at the interface, even at the expense of the generation of a significant amount of structural disorder. Therefore, in addition to results for diamond, Fig. 5 also includes our previously determined values for the interfacial conductance of two (001) twist grain boundaries in $\mathrm{Si}$. These values are close to an order of magnitude lower than for the corresponding diamond GBs, which should not be too surprising since the room-temperature thermal conductivity of bulk $\mathrm{Si}$ is also much less than that of diamond (150 versus $2000 \mathrm{~W} / \mathrm{m} \mathrm{K}$ ). This difference in bulk properties can be taken into account through the Kapitza length, $l_{K}$
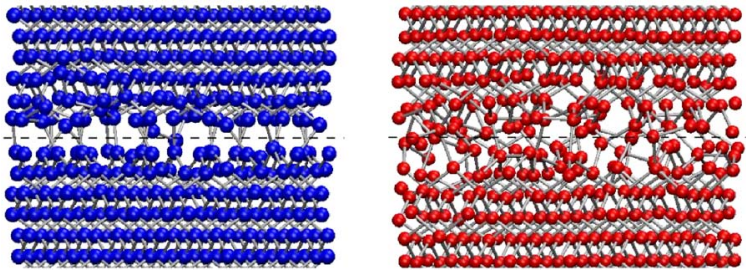

FIG. 7. (Color online) Visualization of $\Sigma 29(001)$ GBs for diamond (left) and $\mathrm{Si}$ (right). Freedom for $\mathrm{C}$ atoms to choose $s p^{2}$ and $s p^{3}$ hybridization results in the rather open structure within the grain boundary. The dashed lines indicate the location of the grain boundaries.

$=\kappa / G_{K}$, which is the thickness of perfect crystal offering the same thermal resistance as the grain boundary. Figure 6 shows the Kapitza length, given in terms of the lattice parameter of the respective materials, as a function of twist angle. We see that due to their lower single-crystal thermal conductivity and their larger lattice parameter, the Si grain boundaries actually have somewhat lower Kapitza lengths, indicating that Si GBs actually allow more efficient heat flow than the corresponding diamond GBs.

To understand the origin of these differences in interfacial conductances, it is necessary to consider the structure of the grain boundaries in more detail. Figure 7 shows edge-on views of the $\Sigma 29$ GBs in both $\mathrm{Si}$ and $\mathrm{C}$. It is evident from the figure, and quantified in Table II, that there is considerably less bonding across the interface in diamond than in silicon. In particular, in diamond, only $14 \%$ of atoms at the GB have two bonds across the interface, while in $\mathrm{Si}, 82 \%$ of atoms have two bonds across the interface. Thus structurally, the Si GB is much better connected. This extra bonding across the $\mathrm{Si}$ interface naturally makes it easier for vibrational excitations, i.e., heat, to cross the interface also.

\section{AN EXTENDED READ-SHOCKLEY MODEL FOR INTERFACIAL THERMAL CONDUCTANCE}

The Read-Shockley (RS) model describes the energies of low-angle GBs in terms of dislocation cores and an associated strain field. ${ }^{19}$ Wolf have made an empirical extension to the RS model-the extended Read-Shockley (ERS) model $^{20}$ - which fits the energies of grain boundaries over the full range of twist angle. For the case of twofold rotational symmetry as on the (001) plane of diamond, the ERS model is given by

$$
E_{\mathrm{GB}}=\left\{\begin{array}{cl}
\sin 2 \theta\left[E_{c}^{<}-E_{s}^{<} \ln (\sin 2 \theta)\right] / b & \text { for } 0^{\circ} \leqslant \theta<45^{\circ} \\
E^{\mathrm{STGB}}+\sin \left(180^{\circ}-2 \theta\right)\left\{E_{c}^{>}-E_{s}^{>} \ln \left[\sin \left(180^{\circ}-2 \theta\right)\right]\right\} / b & \text { for } 45^{\circ} \leqslant \theta<90^{\circ} .
\end{array}\right.
$$

$E_{c}$ and $E_{s}$ are the energies associated with dislocation core and strain field, respectively. $E^{<}$and $E^{>}$are used to indicate the parameters for angles smaller and larger than $45^{\circ}$ twist angle, respectively. $E^{\mathrm{STGB}}$ is the energy of the symmetric twin grain boundatry (STGB) $\left(\theta=90^{\circ}\right) . b$ and $\theta$ are Burger's vector and twist angle, respectively.

Figure 8 shows the calculated GB energy for a large number of (001) diamond twist GBs. (There are many more 
TABLE II. Comparison of the grain boundary energies and coordination of the atoms at the interface in diamond and $\mathrm{Si}$.

\begin{tabular}{ccc}
\hline \hline & Diamond & $\mathrm{Si}$ \\
\hline$E_{\mathrm{GB}}\left(\mathrm{J} / \mathrm{m}^{2}\right)$ & 6.19 & 1.62 \\
$\langle C\rangle$ & 3.11 & 4.02 \\
$C_{2}(\%)$ & 3 & 0 \\
$C_{3}(\%)$ & 83 & 8 \\
$C_{4}(\%)$ & 14 & 82 \\
$C_{5}(\%)$ & 0 & 10 \\
\hline \hline
\end{tabular}

data points in this plot than in the plot for the Kapitza conductance because the GB energy calculations are not computationally very demanding.) The solid line in Fig. 8 is the ERS fit to the data, which quite well reproduces the trend in the energies.

Also shown in Fig. 8 are the contributions to the energy from the dislocation core (dashed line) and the strain field (dash-dot line) as determined from the extended ReadShockley equation. At most twist angles, the structural contribution, which at low angles is due to dislocation cores, dominates the energy. Particularly at $45^{\circ}$ and $90^{\circ}$, there is no strain field contribution. The only boundary condition that restricts the values of these coefficients is the continuity of the two functions at $45^{\circ}$, which yields the constraint, $E_{C}^{<} / b$ $=E_{C}^{>} / b-E^{\mathrm{STGB}} . E_{\mathrm{GB}}$ at $0^{\circ}$, and $d E_{\mathrm{GB}} / d \theta$ at $45^{\circ}$ are 0 by default. The parameters of the ERS model obtained from the fit are

$$
E_{C}^{<} / b=5.94 \mathrm{~J} / \mathrm{m}^{2}, \quad E_{S}^{<} / b=5.24 \mathrm{~J} / \mathrm{m}^{2},
$$

$$
l_{K}=\left\{\begin{array}{c}
\sin 2 \theta\left[l_{c}^{<}-l_{s}^{<} \ln (\sin 2 \theta)\right] \\
l^{\mathrm{STGB}}+\sin \left(180^{\circ}-2 \theta\right)\left\{l_{c}^{>}-l_{s}^{>} \ln \left[\sin \left(180^{\circ}-2 \theta\right)\right]\right\}
\end{array}\right.
$$

for $0^{\circ} \leqslant \theta<45^{\circ}$

for $45^{\circ} \leqslant \theta<90^{\circ}$.

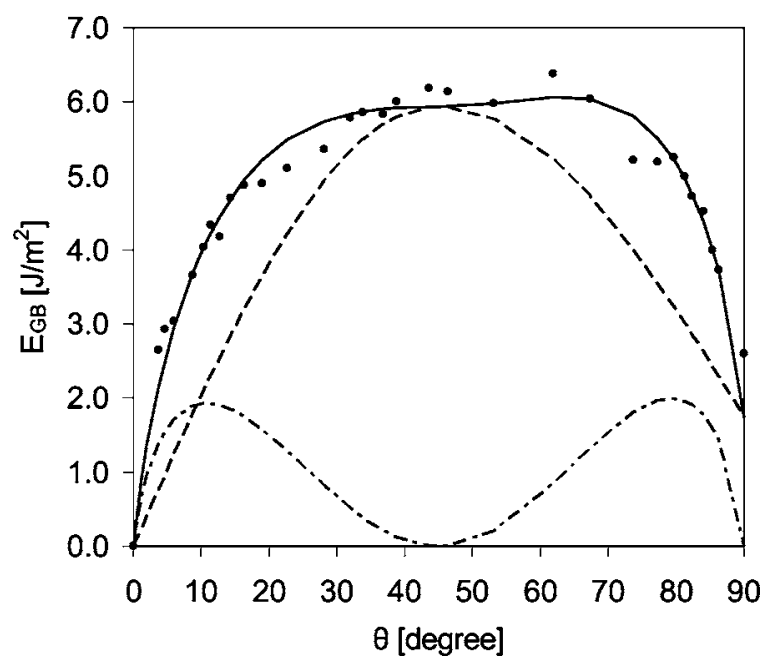

FIG. 8. Grain boundary energy (symbols) and the fit to the extended ReadShockley model (solid line). The dashed line is the dislocation core contribution to the GB energy. The dash-dot line is the strain field contribution.

$$
\begin{aligned}
& E_{C}^{>} / b=4.19 \mathrm{~J} / \mathrm{m}^{2}, \quad E_{S}^{>} / b=5.42 \mathrm{~J} / \mathrm{m}^{2}, \\
& E^{\mathrm{STGB}}=1.75 \mathrm{~J} / \mathrm{m}^{2} .
\end{aligned}
$$

Interestingly, the general trend in the Kapitza length shown in Fig. 6 is similar to that of the GB energy shown in Fig. 8. To show this correlation more clearly, Fig. 9 shows that the Kapitza length depends approximately linearly on the GB energy; the slope of the linear fit is $85.3 \mathrm{~m}^{3} / \mathrm{J}$.

This approximate linear relation indicates that the Kapitza length should also be describable by an ERS model,
The solid line in Fig. 6 is an ERS fit to the Kapitza length. In analogy with the energy of the dislocation core and the strain field energy, it is natural to identify the coefficients of this ERS model as the Kapitza lengths associated with the structural disorder in the GBs and with the strain field.

From the fit obtained from the $E_{\mathrm{GB}}$, we determine the coefficients of $l_{K}$ to be the following:

$$
\begin{aligned}
& l_{C}^{<}=134.3 a_{0}, \quad l_{S}^{<}=105.3 a_{0}, \quad l_{C}^{>}=13.4 a_{0}, \\
& l_{S}^{>}=71.1 a_{0}, \quad l^{\mathrm{STGB}}=121.0 a_{0}
\end{aligned}
$$

As in the case of the energy, the ERS form forces the condition $l^{\mathrm{STGB}}+l_{C}^{>}=l_{C}^{<}$by continuity. The strain contributions to the Kapitza length differ by $\sim 30 \%$ in contrast to the corresponding values for the energy that differ by only $5 \%$.

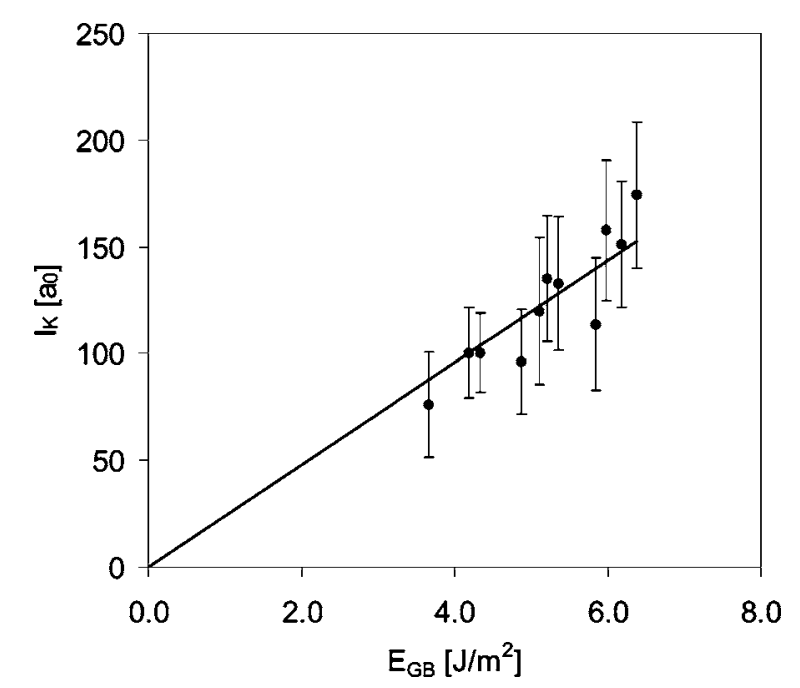

FIG. 9. The Kapitza length is a reasonably linear function of the grain boundary energy. 


\section{DISCUSSION}

The work presented here has shown that the interfacial (Kapitza) resistance of (001) twist grain boundaries in diamond depends systematically on the twist angle. Importantly, the Kapitza length is proportional to the energy of the grain boundary, allowing us to fit it with an extended ReadShockley model.

These results suggest that the energy of a grain boundary, which is easily calculated, might be used as a surrogate quantity for the interfacial conductance, which is more difficult and computationally very expensive to determine. Further work on different grain boundaries in diamond, and on different materials systems, is required to establish if this is a general behavior or if it is particular to this system.

Regardless of the specific details, however, this work does suggest that it should be possible to determine an empirical relationship for the interfacial conductance as a function of the crystallography of the material. Such a relationship will be of considerable value as input to mesoscale simulations of thermal transport.

\section{ACKNOWLEDGMENTS}

We would like to thank Jasmine Davenport for her assistance with some of the simulations. The work of T.W. and S.R.P. was supported by DOE-NERI Award No. DE-FC0705ID14649. The work of B.N. was supported by the Ameri- can Chemical Society Petroleum Research Fund.

${ }^{1}$ P. K. Schelling, L. Shi, and K. E. Goodson, Mater. Today 8, 30 (2005).

${ }^{2}$ D. G. Cahill, W. K. Ford, K. E. Goodson, G. D. Mahan, A. Majumdar, H. J. Maris, R. Merlin, and S. R. Phillpot, J. Appl. Phys. 93, 793 (2003).

${ }^{3}$ B. Becker, P. K. Schelling, and S. R. Phillpot, J. Appl. Phys. 99, 123715 (2006).

${ }^{4}$ Y. F. Chen, D. Y. Li, J. K. Yang, Y. H. Wu, J. R. Lukes, and A. Majumdar, Physica B 349, 270 (2004).

${ }^{5}$ P. K. Schelling, S. R. Phillpot, and P. Keblinski, J. Appl. Phys. 95, 6082 (2004).

${ }^{6}$ H. O. Pierson, Handbook of Carbon, Graphite, Diamond and Fullerenes: Properties, Processing and Applications (Noyes, Park Ridge, NJ, 1993).

${ }^{7}$ R. F. Davis, Diamond Films and Coatings (Noyes, Park Ridge, NJ, 1993).

${ }^{8}$ M. H. Nazare and A. J. Neves, Properties, Growth and Applications of Diamond (Institution of Engineering and Technology, London, 2001).

${ }^{9}$ O. Auciello, J. Birrell, J. A. Carlisle, J. E. Gerbi, X. C. Xiao, B. Peng, and

H. D. Espinosa, J. Phys.: Condens. Matter 16, R539 (2004).

${ }^{10}$ J. Tersoff, Phys. Rev. Lett. 61, 2879 (1988).

${ }^{11}$ P. Jund and R. Jullien, Phys. Rev. B 59, 13707 (1999).

${ }^{12}$ P. K. Schelling, S. R. Phillpot, and P. Keblinski, Phys. Rev. B 65, 144306 (2002).

${ }^{13}$ P. Keblinski, D. Wolf, S. R. Phillpot, and H. Gleiter, J. Mater. Res. 13, 2077 (1998).

${ }^{14}$ S. von Alfthan, P. D. Haynes, K. Kaski, and A. P. Sutton, Phys. Rev. Lett. 96, 055505 (2006).

${ }^{15}$ T. Watanabe, P. Shukla, and S. R. Phillpot, submitted to J. Nucl. Mater.

${ }^{16}$ R. J. Stoner and H. J. Maris, Phys. Rev. B 48, 16373 (1993).

${ }^{17}$ H. K. Lyeo and D. G. Cahill, Phys. Rev. B 73, 144301 (2006).

${ }^{18}$ R. J. Stevens, L. V. Zhigilei, and P. M. Norris, Int. J. Heat Mass Transfer 50, 3977 (2007).

${ }^{19}$ W. Shockley and W. T. Read, Phys. Rev. 75, 692 (1949).

${ }^{20}$ D. Wolf, Scripta Metallurgica 23, 1713-1718 (1989). 